Original research article

\title{
The frequency and consequences of multipolar mitoses in undifferentiated embryonic stem cells
}

\author{
Veronika Pospíšilová ${ }^{1}$, Milan Ešner ${ }^{1,2}$, Iveta Červenková ${ }^{1}$, Radek Fedr ${ }^{4}$, Jean-Yvez Tinevez ${ }^{5}$, \\ Aleš Hampl ${ }^{1,3}$, Martin Anger ${ }^{1,2}$ * \\ ${ }^{1}$ Masaryk University, Faculty of Medicine, Department of Histology and Embryology, Brno, Czech Republic \\ ${ }^{2}$ Masaryk University, CEITEC - Central European Institute of Technology, Cellular Imaging Core Facility, Brno, Czech Republic \\ ${ }^{3}$ St. Anne's University Hospital, International Clinical Research Center, Brno, Czech Republic \\ ${ }^{4}$ Institute of Biophysics of the Czech Academy of Sciences, Brno, Czech Republic \\ ${ }^{5}$ Pasteur Institute, Image Analysis Hub, Paris, France
}

\section{Abstract}

Embryonic stem (ES) cells are pluripotent cells widely used in cell therapy and tissue engineering. However, the broader clinical applications of ES cells are limited by their genomic instability and karyotypic abnormalities. Thus, understanding the mechanisms underlying ES cell karyotypic abnormalities is critical to optimizing their clinical use. In this study, we focused on proliferating human and mouse ES cells undergoing multipolar divisions. Specifically, we analyzed the frequency and outcomes of such divisions using a combination of time-lapse microscopy and cell tracking. This revealed that cells resulting from multipolar divisions were not only viable, but they also frequently underwent subsequent cell divisions. Our novel data also showed that in human and mouse ES cells, multipolar spindles allowed more robust escape from chromosome segregation control mechanisms than bipolar spindles. Considering the frequency of multipolar divisions in proliferating ES cells, it is conceivable that cell division errors underlie ES cell karyotypic instability.
\end{abstract}

Keywords: Embryonic stem (ES) cells; Mitosis length; Multipolar division; Single-cell tracking; Spindle assembly checkpoint (SAC); Time-lapse microscopy

Highlights:

- Undifferentiated embryonic stem (ES) cells that undergo multipolar divisions are viable and continue to divide.

- Polyploid ES cells generated by inhibition of cytokinesis more frequently undergo multipolar division.

- Cells undergoing multipolar division escape from spindle assembly checkpoint (SAC) activity faster than bipolar dividing cells.

\section{Introduction}

Embryonic stem (ES) cells are pluripotent cells derived from the inner cell mass of the blastocyst (Martello and Smith, 2014). Because ES cells can differentiate into every cell type in the human body, they are potentially an ultimate source material for regenerative medicine. However, the use of ES cells for such applications is limited by their chromosomal instability and frequent acquisition of karyotypic abnormalities, including chromosomal translocations and aneuploidy, during propagation in vitro (Draper et al., 2004; Imreh et al., 2006; International Stem Cell Initiative et al., 2011; Spits et al., 2008). Thus, understanding the mechanisms underlying karyotypic abnormalities in ES cells is critical to optimizing their medical use.

Several studies of cancer cells have shown that aneuploidy might result from errors in spindle assembly caused by the presence of supernumerary centrosomes (Brinkley, 2001). Indeed, cells containing supernumerary centrosomes can undergo multipolar divisions, which result in anaphase catastrophe and cell death (Brinkley, 2001; Castedo et al., 2004; Galimberti et al., 2011; Vitale et al., 2011). Alternatively, other studies have reported that even in the presence of supernumerary centrosomes, cells can prevent multipolar spindle assembly and the associated consequences. Specifically, cells can inactivate extra centrosomes or cluster several centrosomes into one spindle pole (Quintyne et al., 2005; Ring et al., 1982). Cells with supernumerary centrosomes may also form multipolar spindles only transiently but ultimately divide with a bipolar spindle (Ganem et al., 2009). Correspondingly, centrosome (de)clustering mechanisms have become a key component of potential cancer therapies. Indeed, researchers are now searching for molecules that can inhibit centrosome clustering and induce mitotic/anaphase catastrophe by multipolar division (Galimberti et al., 2011; Kwon et al., 2008).

\footnotetext{
* Author for correspondence: Martin Anger, Kamenice 3, Building A1, 62500 Brno, Czech Republic; e-mail: anger@med.muni.cz http://doi.org/10.32725/jab.2019.018

Submitted: 2019-05-31 • Accepted: 2019-10-22 • Prepublished online: 2019-11-11

J Appl Biomed 17/4: 209-217 • EISSN 1214-0287 • ISSN 1214-021X

(C) 2019 The Authors. Published by University of South Bohemia in České Budějovice, Faculty of Health and Social Sciences.

This is an open access article under the CC BY-NC-ND license.
} 
Similar to cancer cells, human ES cells frequently harbor extra centrosomes (Holubcova et al., 2011). However, whether a link between supernumerary centrosomes and multipolar spindles exists for ES cells is unclear. Chromosome segregation errors can be prevented by a control mechanism called the spindle assembly checkpoint (SAC). SAC prevents anaphase onset in the presence of unattached or improperly attached chromosomes and arrests mitosis until all sister kinetochores are properly connected to microtubules (Foley and Kapoor, 2013). In cancer cells, SAC allows sufficient time for centrosome clustering, thereby preventing multipolar spindle formation prior to anaphase (Kwon et al., 2008). Although SAC is intact in ES cells (Ballabeni et al., 2011), the fine details of its operation remain unknown.

In this study, we focused on multipolar divisions in proliferating human and mouse ES cells. Specifically, we evaluated the frequency of multipolar mitoses in live cells, providing the first quantification of this phenomenon during proliferation cycles in undifferentiated cells. Our data showed that ES cells frequently underwent multipolar mitoses during propagation in vitro. We then used cell tracking to study the fate of cells resulting from such divisions. This revealed that not only were the daughter cells viable for a relatively long time in culture, but they also frequently underwent subsequent divisions. Furthermore, the frequency of multipolar mitoses increased with polyploidization, which led to centrosome duplication. We also showed for the first time that following SAC activation induced by low levels of nocodazole, ES cells with multipolar spindles divided faster than those with bipolar spindles. Thus, for the therapeutic applications of ES cells, multipolar divisions are a major concern, primarily due to the limited effectiveness of chromosome segregation control mechanisms and the survival potential of such cells.

\section{Materials and methods}

\section{DNA constructs}

Bacterial artificial chromosome (BAC) \#3715 containing H2A tagged with EGFP, which was kindly donated by A. Hyman (MPI-CBG, Dresden, Germany), was used to transfect human ES cells. For mouse ES cells, we used PCR to amplify cDNA H2B-mCherry from the pRNA His-mCherry vector with the following primers: 5'-TCAAGCTTACCATGGCTAGCCCTGAACTGGCCAAATCTGCCCCG-3' and 5'-TAGTCGACGGCATGGACGAGCTGTACAAGTAA-3'. The mCherry-coding sequence was removed from expression vector $\mathrm{pEF} 1 \alpha$-mCherry-N1 (Cat. \# 631969; Clontech, Kusatsu, Japan) by digestion with HindIII and Sall. These same restriction enzymes were then used to insert PCR amplicons. Before transfection, all vectors were verified by sequencing.

\section{Immunocytochemistry}

Cells were fixed with $4 \%$ formaldehyde and permeabilized with $0.1 \%$ Triton X-100 (Carl Roth, Karlsruhe, Germany) in phosphate-buffered saline (PBS) for $10 \mathrm{~min}$ at room temperature (RT). Primary antibody was diluted in $1 \%$ bovine serum albumin (BSA; Sigma-Aldrich, St. Louis, MO) in PBS containing $0.05 \%$ Tween-20 (Sigma-Aldrich) and then incubated for $60 \mathrm{~min}$ at RT (CREST antibody was incubated overnight at $4{ }^{\circ} \mathrm{C}$ ). After washing with PBS, secondary antibodies conjugated with Alexa Fluor 488, 568, and 594, respectively, were applied for $60 \mathrm{~min}$ at RT (Thermo Fisher Scientific, Waltham, MA). Nuclei were labeled with Hoechst-33342 solution (H3570; Life Technologies, Carlsbad, CA) diluted to $2 \mu \mathrm{g} / \mathrm{ml}$ in
PBS for the last washing step and then samples were mounted with Mowiol. Primary antibodies used were as follows: pericentrin (ab4448, 1 : 8,000; Abcam, Cambridge, UK), OCT-4 (SAB105A-1, 1:100; System Biosciences, Palo Alto, CA), and human nuclear ANA-Centromere autoantibody CREST (CS1058, 1 : 1,000; Europa Bioproducts, Wicken, UK).

\section{Cell lines and cell culture}

Transgenic human ES cell lines were produced from the parental line CCTL14 and routinely maintained in the laboratory (https://hpscreg.eu/cell-line/MUNIe007-A). Undifferentiated human ES cells were maintained on $\gamma$-irradiated mouse fibroblasts (MEFs) as described previously (Holubcova et al., 2011).

Cells of the JM8.A line of mouse ES cells, donated by Ronald Naumann (MPI-CBG, Dresden, Germany), were cultured on gelatin-coated dishes in knockout Dulbecco's modified Eagle medium (DMEM) with high glucose and supplemented with $2 \mathrm{mM}$ GlutaMax, $1 \mathrm{mM}$ NE Amino Acids (Thermo Fisher), $1 \mu \mathrm{M}$ 2-Mercaptoethanol (Sigma-Aldrich), 15\% fetal bovine serum (FBS; Thermo Fisher), and 1,000 U/ml leukemia inhibitory factor (LIF; Thermo Fisher). Mouse ES cells were transfected with Lipofectamine 3000 (Thermo Fisher) according to the manufacturer's instructions. Positive clones were selected using G418 antibiotic and sorted using fluorescence-activated cell sorting (FACS).

Transfection and selection of human ES cells were performed in feeder-free conditions on dishes coated with Geltrex (Cat. \# A1413301; Thermo Fisher) in human ES cell medium preconditioned with MEFs. Undifferentiated human ES cells were transfected with FugeneHD (Promega, Madison, WI) according to the manufacturer's instructions. After transfection, positive cells were selected using G418 antibiotic and sorted using FACS. After establishing stability, cells were further cultured on feeder cells without G418 as described previously (Poser et al., 2008). Positive clones were first characterized for expression of pluripotency markers and the percentage of centrosomal abnormalities and then further expanded (data not shown).

Polyploidy was induced by exposure of unsynchronized human/mouse ES cells to cytochalasin D (C8273; Sigma-Aldrich) for $12 \mathrm{~h}$ to prevent faithful cytokinesis. Cytochalasin D was used at a final concentration of $0.5 \mu \mathrm{M}$ by diluting $2000 \times$ dimethyl sulfoxide (DMSO) stock. Drug treatment was terminated by washing with drug-free medium five times over $30 \mathrm{~min}$. Subsequent counting of bipolar/multipolar mitoses was performed only for divisions in which mitotic spindles formed from two nuclei.

Nocodazole (M1404; Sigma-Aldrich) was used as antimitotic agent for SAC challenge. Nocodazole was stored at a concentration of $10 \mathrm{mM}$ in DMSO and then used at final concentrations of $25 \mathrm{nM}, 50 \mathrm{nM}$, or $100 \mathrm{nM}$, respectively, depending on the experiment. The maximal concentration of DMSO in culture media was $0.0001 \%$. Nocodazole was maintained in culture media throughout the experiment.

\section{Microscopy, live-cell imaging, and cell tracking}

For live-cell imaging experiments, DMEM/F12 without phenol red and supplemented with 25 mM HEPES was used (Thermo Fisher). Medium in 96-well plates was overlaid with $50 \mu \mathrm{l}$ of mineral oil (M8410; Sigma-Aldrich) to prevent evaporation. Time-lapse movies were acquired using an ImageXpress Micro XL automated epifluorescence microscope (Molecular Devices, San Jose, CA) with the $40 \times / 0.6$ objective and the chamber preheated to $37{ }^{\circ} \mathrm{C}$. The frame rate was one image every 15 min. Images of fixed cells were acquired with a Nikon Ti 
Eclipse inverted fluorescence microscope using the $100 \times / 1.45$ objective and a Nikon DS-Qi2 camera or a Zeiss LSM 780 confocal microscope using the Plan-Apochromat $63 \times / 1.4$ objective, respectively.

For cell tracking, we used the TrackMate plugin inside the FIJI open-source platform adapted to count multipolar mitoses. Fixed cells were analyzed using the Cell Profiler open-source software (www.cellprofiler.org). Data were analyzed and plots were produced using $\mathrm{R}$ software for statistical computing and graphics (https://cran.r-project.org/) with the ggplot2 package.

\section{Statistical analysis}

All statistical tests were performed using non-parametric Kruskal-Wallis tests in open-source R software (https:// cran.r-project.org/).

\section{Results}

\section{Frequency of multipolar divisions in dividing human ES cells}

As we have published previously, human ES cells suffer from supernumerary centrosomes (Holubcova et al., 2011), which is associated with the development of multipolar spindles. To evaluate the frequency of multipolar divisions in undifferentiated human ES cells, we visualized their chromatin using expression of a BAC transgene encoding histone $\mathrm{H} 2 \mathrm{~A}$ fused to EGFP. Live cells were recorded for $72 \mathrm{~h}$ and every $15 \mathrm{~min}$, a single $z$-plane image was acquired at four independent positions in each well. Based on the division geometry, cells were divided into two categories: those with bipolar division, which yielded two daughter cells, and those with multipolar division, which yielded more than two daughter cells (Fig. 1A). For these time-lapse experiments, a total of 21,538 mitoses were scored, of which 557 divisions (2.7\%) were multipolar. Because the percentage of multicentrosomal cells changes with the passage number in human ES cells, we could not directly correlate the number of multipolar divisions with the occurrence of multicentrosomal cells from previous findings (Holubcova et al., 2011). Instead, we visualized centrosomes in fixed cells at the same passage number and then performed an indirect correlation between the number of multicentrosomal mitotic spindles (Fig. 1B) and the frequency of multipolar mitotic divisions. This showed that the frequency of multipolar divisions was comparable to the frequency of cells with supernumerary centrosomes, suggesting that no mechanisms would lead to centrosome clustering and pseudo-bipolar division (Fig. 1C).

A
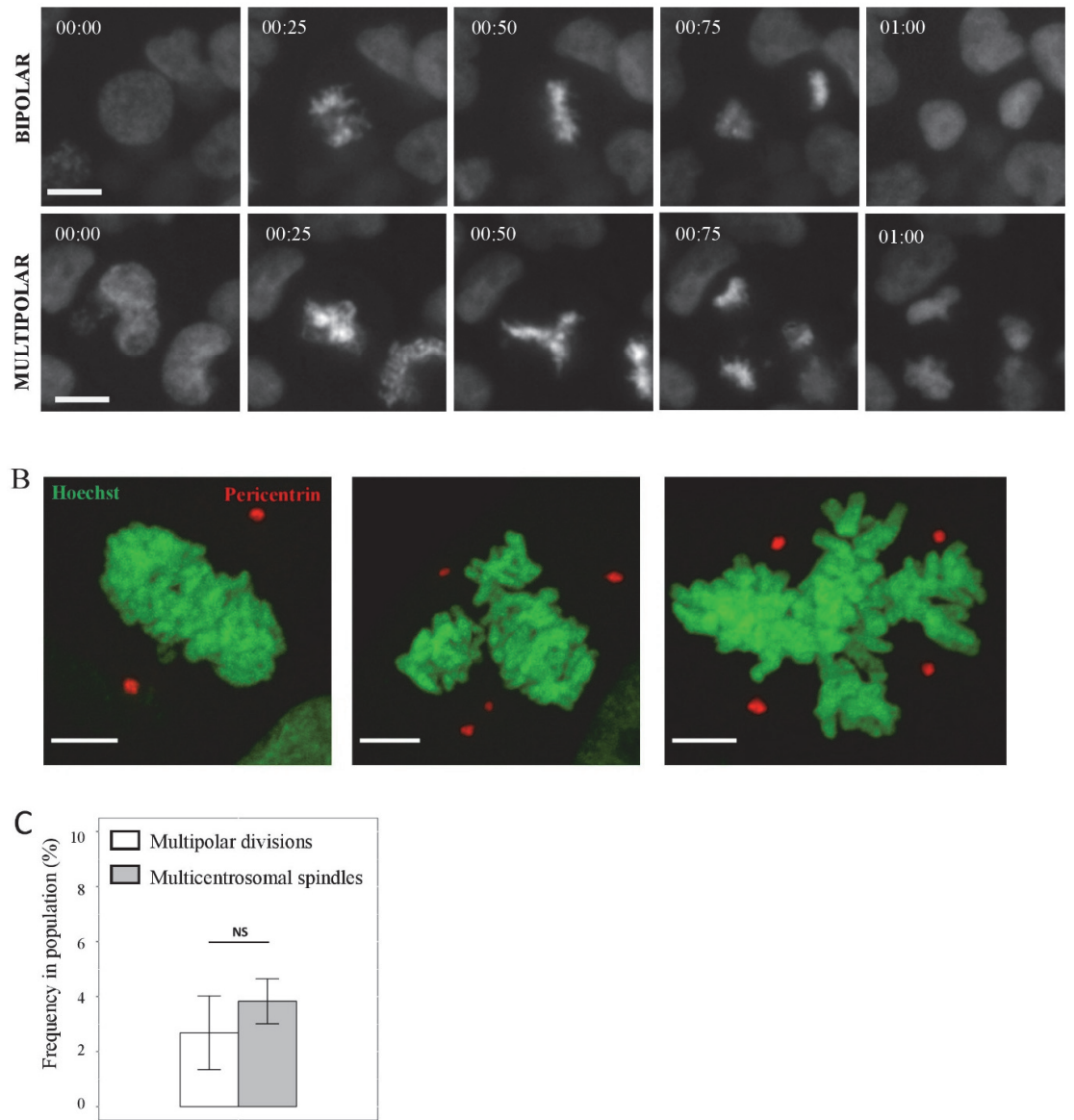

Fig. 1. The frequency of multipolar divisions and supernumerary centrosomes in undifferentiated human embryonic stem (ES) cells. (A) Frames from the time-lapse imaging experiment showing cells undergoing bipolar (top) and multipolar divisions (bottom). DNA was visualized by expression of Histone H2A fused to EGFP. Scale bar $=10 \mu \mathrm{m}$, relative time is indicated. (B) Examples of human ES cells with two centrosomes (left) and supernumerary centrosomes (middle and right). Centrosomes were visualized using an anti-pericentrin antibody (red) and chromatin with Hoechst (green). Scale bar $=5 \mu \mathrm{m}$. (C) Chart comparing the frequency of multipolar divisions (white bar) scored from live cells in time-lapse experiments and the frequency of cells with supernumerary centrosomes (gray bar) as analyzed using immunofluorescence in fixed cells from the same experiments. Data were collected in three experiments. The total number of cells analyzed for multipolar divisions was 21538 and the total number of cells analyzed for the number of centrosomes was 2840. Error bars represent standard deviation. Differences between conditions were evaluated by Kruskal-Wallis test: not significant (n.s.) $p>0.05,{ }^{*} p \leq 0.05,{ }^{* *} p \leq 0.01,{ }^{* * *} p \leq 0.001,{ }^{* * * *} p \leq 0.0001$. 


\section{Cell fates after multipolar divisions}

To evaluate the impact of multipolar divisions on applications of ES cells in cell engineering and therapy, we next evaluated the fate of cells resulting from such divisions. This information is crucial because these cells are obviously aneuploid and therefore might limit clinical applicability. To evaluate this, we used the TrackMate plugin in the FIJI open-source software, which was modified to detect multipolar divisions. We used the same dataset as in Fig. 1 for this analysis. Individual cells and their progeny were tracked in images acquired during time-lapse experiments (Fig. 2A).

Of the 557 multipolar divisions observed, the vast majority $(545 ; 97.8 \%)$ produced viable progeny. In principle, these cells would carry chromosomal abnormalities, so it was important to determine their fate. To do this, we used single-cell tracking to further analyze these cells. First, we evaluated the viability of cells resulting from multipolar divisions. In total, we tracked 129 multipolar divisions, which produced a total of 396 daughter cells. Of these daughter cells, 58 (14.6\%) died immediately after completing mitosis, while the remaining 338 were viable until the end of the experiment. Of the 129 total divisions, only 8 (6.2\%) did not produce any viable offspring.

Surprisingly, our data showed that cells undergoing multipolar division spent a significantly longer time in preceding interphase than those undergoing bipolar division
(Fig. 2B). Specifically, cells undergoing bipolar division had an average interphase length preceding mitosis of approximately $19 \mathrm{~h}$, while those undergoing multipolar division had an average of $27 \mathrm{~h}$. Furthermore, some neighboring nuclei combined into one mitotic spindle, suggesting the existence of binuclear cells. As our cells did not carry membrane markers, we were unable to determine whether this was due to cell fusion or cytokinesis failure. In some cases, we observed this phenomenon in cells originating from multipolar divisions (Fig. 2A).

\section{The impact of polyploidization on multipolar mitosis frequency in ES cells}

One possible mechanism underlying the production of binuclear cells is cytokinesis inhibition and subsequent formation of binuclear polyploid cells. During polyploidization, cells accumulate extra centrosomes (Gentric and Desdouets, 2014; Storchova and Kuffer, 2008). A previous study from our laboratory showed that a significant proportion of human ES cells with supernumerary centrosomes are tetraploid (Holubcova et al., 2011). To study the relationship between polyploidy and multipolar division, we treated cells with cytochalasin D to inhibit cytokinesis and enrich the percentage of binuclear/ polyploid cells with extra centrosomes (Fig. 3A). For all subsequent experiments, we evaluated both human and mouse ES cells to ensure that our results were not limited to one species. By using cytochalasin $\mathrm{D}$, we were able to more frequently
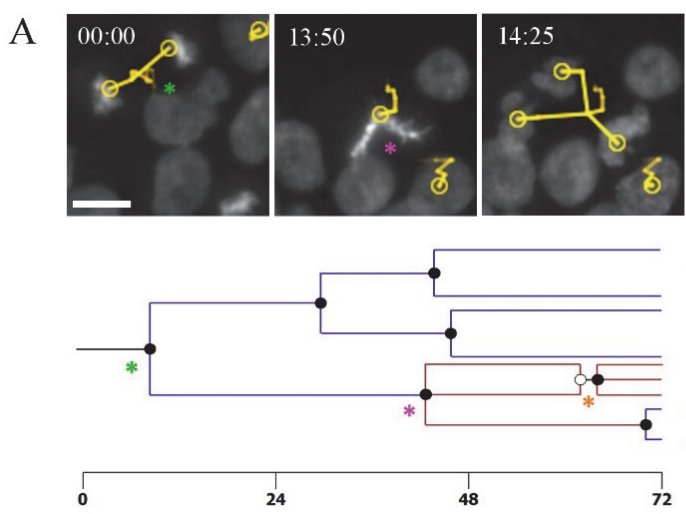

24

B

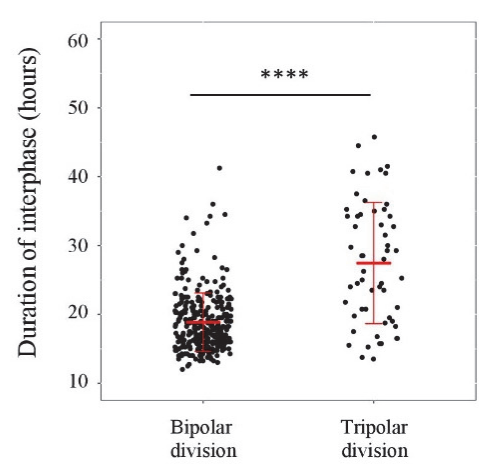

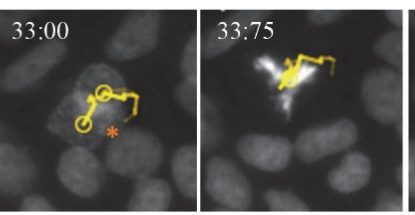

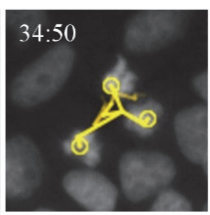

Mitotic spindle from

one nucleus

Mitotic spindle from

two nuclei

- Bipolar division

- Multipolar division 
A

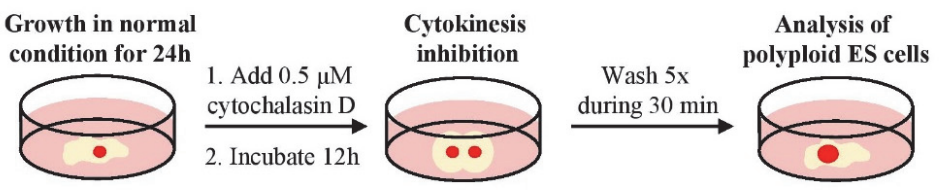

B

$\mathrm{C}$

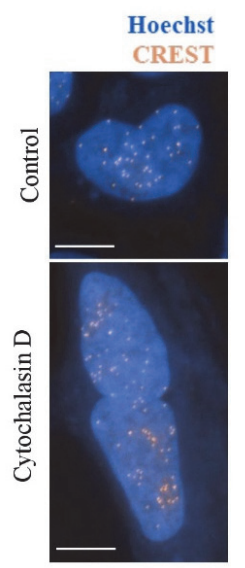

\begin{tabular}{|l|c|c|c|c|c|c|c|c|}
\hline & \multicolumn{4}{|c|}{ Human } & \multicolumn{4}{c|}{ Mouse } \\
\cline { 2 - 8 } & \multicolumn{2}{|c|}{ Control } & \multicolumn{2}{c|}{ Cytochalasin D } & \multicolumn{2}{c|}{ Control } & \multicolumn{2}{c|}{ Cytochalasin D } \\
\hline Division type & Count & $\begin{array}{c}\% \text { of } \\
\text { total }\end{array}$ & Count & $\begin{array}{c}\% \text { of } \\
\text { total }\end{array}$ & Count & $\begin{array}{c}\% \text { of } \\
\text { total }\end{array}$ & Count & $\begin{array}{c}\% \text { of } \\
\text { total }\end{array}$ \\
\hline Bipolar & 4605 & 98,75 & 2613 & 71,65 & 896 & 99,78 & 1062 & 93,04 \\
Multipolar & 58 & 1,24 & 1034 & 28,35 & 2 & 0,22 & 87 & 7,57 \\
\hline
\end{tabular}

Fig. 3. Cytochalasin D-induced cytokinesis inhibition induces polyploidy and increases the frequency of multipolar divisions in human and mouse ES cells. (A) Experimental overview: ES cells from both species were exposed to $0.5 \mu \mathrm{M}$ cytochalasin D for $12 \mathrm{~h}$. Thirty minutes after removal of the inhibitor from the media, cells were analyzed by live-cell imaging or by immunocytochemistry. (B) Representative images of the control human ES cells (top) and cells containing neighboring nuclei after cytochalasin D treatment. Kinetochores (orange) were visualized using CREST antibody and DNA (blue) was labeled with Hoechst. Scale bar $=10 \mu \mathrm{m}$. (C) The frequency of bipolar and multipolar divisions scored from the time-lapse experiments after cytochalasin D-induced polyploidy. The number of divisions scored in each category is indicated.

obtain cells with two nuclei and with the number of kinetochores consistent with polyploidy (Fig. 3B). Subsequently, we evaluated the frequency of multipolar divisions in human and mouse ES cells treated with cytochalasin D. This revealed that cytochalasin $\mathrm{D}$ increased the frequency of multipolar divisions in ES cells from both species by more than 20 times, showing that induction of binuclear/polyploid cells leads to multipolar division (Fig. 3C).

\section{Nocodazole dose-dependently affects proliferation and mitosis length in ES cells}

Multipolar spindle formation, even transiently, challenges the fidelity of chromosome segregation (Silkworth and Cimini, 2012; Silkworth et al., 2009). However, whether multipolar spindles are detected by control mechanisms such as SAC is unclear. Some studies have shown that cells with multipolar spindles exhibit a metaphase-anaphase delay, which is abolished by targeting SAC (Kwon et al., 2008). Others have shown that in cancer cells, Mad1 signaling is absent from kinetochores in a large portion of cells with multipolar spindles (Gisselsson et al., 2010). Here, we first depolymerized microtubules using nocodazole to determine whether human and mouse ES cells had intact SAC responses. Cells were grown for $24 \mathrm{~h}$ in media containing $25 \mathrm{nM}, 50 \mathrm{nM}$, or $100 \mathrm{nM}$ nocodazole. Because exposing cells to nocodazole might also completely block cell proliferation and induce cell death (Sanchez-Aguilera et al., 2006), we measured cell proliferation by counting nuclei before and after the 24-h nocodazole exposure (Fig. 4A). This revealed that the highest concentration of nocodazole (100 nM) completely prevented any increase in cell number. This was consistent with previous findings made by Kallas et al. using the human ES cell line H9. These results strongly indicated that in ES cells, $25 \mathrm{nM}$ and $50 \mathrm{nM}$ nocodazole genuinely activat- ed SAC (with slower progression through mitosis) rather than causing irreparable cell damage (Kallas et al., 2011).

We then measured how $25 \mathrm{nM}, 50 \mathrm{nM}$, or $100 \mathrm{nM}$ nocodazole treatment affected mitosis length, as prolonged mitosis usually reflects the extension of SAC activity (Fig. 4B). This showed that while human ES cells had increased mitosis length upon exposure to $25 \mathrm{nM}$ nocodazole, mouse ES cells required $50 \mathrm{nM}$ nocodazole to show a similar effect. The average length of mitosis, from nuclear envelope breakdown (NEBD) to cytokinesis, in control human ES cells was $0.6 \pm 0.3 \mathrm{~h}$, while treatment with $25 \mathrm{nM}, 50 \mathrm{nM}$, and $100 \mathrm{nM}$ nocodazole increased this interval to $0.9 \pm 0.7,2.5 \pm 1.5$, and $3.9 \pm 1.5 \mathrm{~h}$, respectively. Similarly, the average length of mitosis in control mouse ES cells was $0.5 \pm 0.1 \mathrm{~h}$, while treatment with $25 \mathrm{nM}$, $50 \mathrm{nM}$, and $100 \mathrm{nM}$ nocodazole extended mitosis to $0.5 \pm 0.2$, $0.7 \pm 0.2$, and $3.1 \pm 0.9 \mathrm{~h}$, respectively (Fig. 4B).

\section{Cells undergoing multipolar division escape from SAC activity faster}

As demonstrated above, nocodazole activated SAC in ES cells. This allowed us to directly address how bi- and multipolar dividing cells reacted to SAC activation. For all subsequent experiments, we used $50 \mathrm{nM}$ nocodazole, as this concentration significantly prolonged mitosis in both species. Furthermore, prior to nocodazole exposure, we enriched the population of cells undergoing multipolar mitosis using cytochalasin D to induce polyploidization (Fig. 5A). We then measured mitosis length using live-cell imaging, and only cells with two nuclei that eventually formed a single mitotic spindle were analyzed (Fig. 5B). This revealed that in control polyploid cells without nocodazole, the mitosis length from NEBD to cytokinesis was $1.3 \pm 0.9 \mathrm{~h}$ for bipolar spindles and $1.1 \pm 0.4 \mathrm{~h}$ for multipolar spindles in human ES cells. For mouse ES cells, this length was 
A

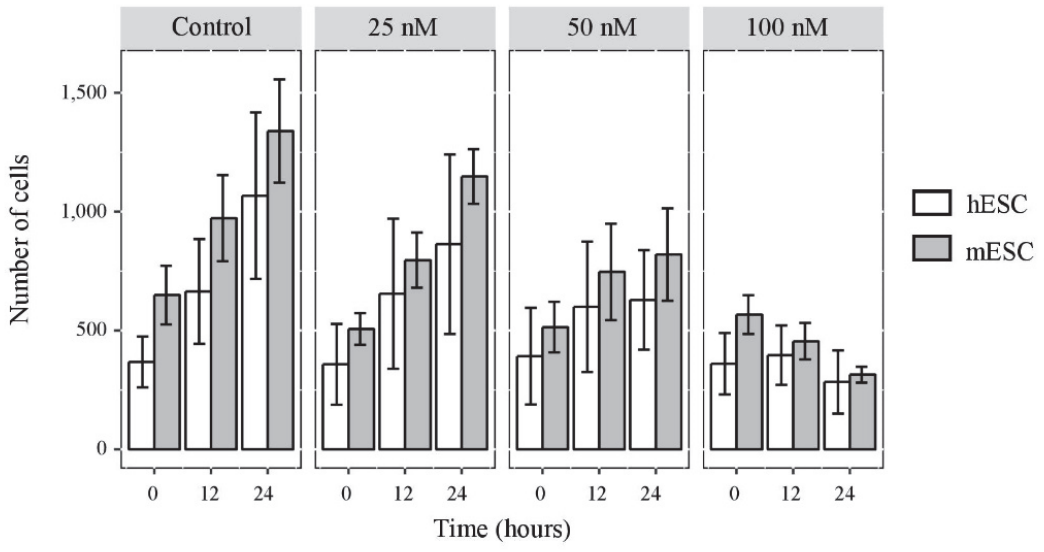

B

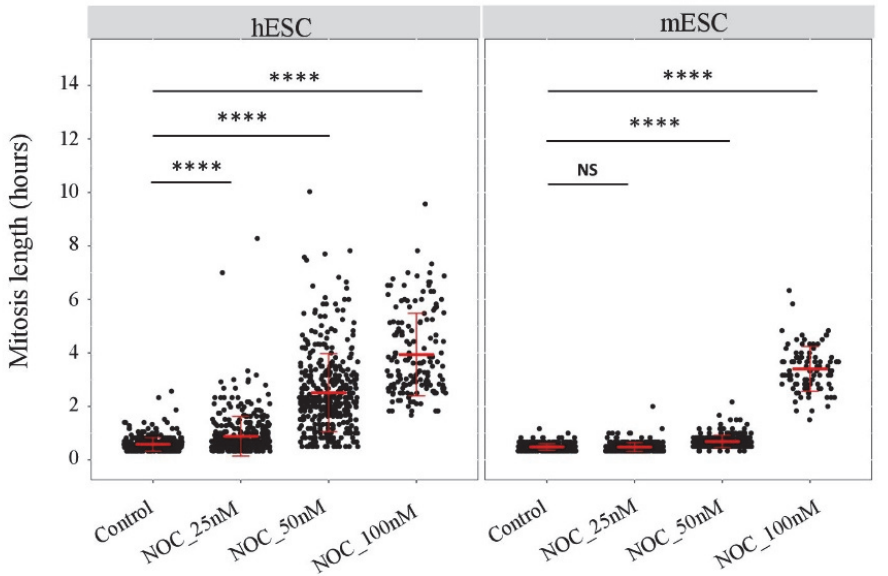

Fig. 4. Nocodazole dose-dependently affects cell proliferation and mitosis length in human and mouse ES cells. (A) Human (white bars) and mouse (gray bars) ES cells were cultured in the presence of different concentrations of nocodazole. Cell counts were measured at 0, 12, and 24 h after initiation of the experiment. Bars represent the mean number of cells in each category and error bars represent the standard error of the mean. Data were obtained from three independent experiments $(n=3)$. (B) The time interval between nuclear envelope breakdown (NEBD) and cytokinesis scored in human (left) and mouse (right) ES cells after nocodazole exposure. Dot plots indicate the mitosis length in each category. Data were collected from three independent experiments and only bipolar mitoses were scored. Statistical values are summarized by plotting mean with error bars representing the standard deviation (red). Differences between conditions were evaluated by Kruskal-Wallis test: n.s. $p>0.05,{ }^{*} p \leq 0.05,{ }^{* *} p \leq 0.01,{ }^{* * *} p \leq 0.001,{ }^{* * * *} p \leq 0.0001$.

$0.9 \pm 0.4 \mathrm{~h}$ for bipolar spindles and $1.1 \pm 0.4 \mathrm{~h}$ for multipolar spindles (Fig. 5C). Importantly, while the mitosis length did not significantly differ between bipolar and multipolar cells for human polyploid ES cells, multipolar cells required significantly more time to complete mitosis for mouse ES cells; however, this difference was very small. As expected, $50 \mathrm{nM}$ nocodazole delayed anaphase onset in cells from both species. In human ES cells, this treatment also prolonged the mitosis length to $3.5 \pm 1.7 \mathrm{~h}$ in bipolar dividing cells and to $2.1 \pm 1.0 \mathrm{~h}$ in multipolar cells. Similarly, in mouse ES cells, this was prolonged to $2.1 \pm 1.0 \mathrm{~h}$ for bipolar dividing cells and $1.9 \pm 0.8 \mathrm{~h}$ for multipolar cells (Fig. 5C). Thus, ES cells in both species escaped nocodazole-activated SAC faster when undergoing multipolar divisions. To our knowledge, this is the first report to show that multipolar division helps cells escape from SAC-induced metaphase arrest.

\section{Discussion}

Several previous studies have shown that pluripotent cells are more frequently affected by chromosome segregation er- rors than other somatic cells (Draper et al., 2004; Imreh et al., 2006; International Stem Cell Initiative et al., 2011; Spits et al., 2008). Furthermore, aneuploidy and other chromosomal aberrations, as well as epigenetic changes, might promote a phenotype closely resembling cancer cells (Werbowetski-Ogilvie et al., 2009). In somatic cells, aneuploidy negatively affects proliferation, yielding an unbalanced genome with important consequences for essential cellular processes (Durrbaum and Storchova, 2016). Alternatively, some somatic cell types such as hepatocytes are prone to aneuploidy without experiencing negative functional impacts (Duncan et al., 2010). Similar to our current results, most hepatocytes acquire polyploidy via cytokinesis failure (Celton-Morizur et al., 2009). Aneuploid cells might initiate steps toward senescence, leading to their elimination by the immune system (Santaguida et al., 2017). In contrast, mouse ES cells carrying an extra copy of a particular chromosome might show an accelerated proliferation rate as long as they are undifferentiated (Zhang et al., 2016). Therefore, to optimize the therapeutic potential of ES cells, it is essential not only to identify the pathways that lead to aneuploidy, but also to evaluate the risks resulting from the presence and persistence of aneuploid cells. 
A

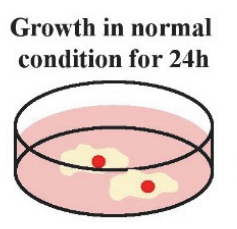

Evaluation of

SAC challenge in polyploid ES cells

1. Wash $5 \mathrm{x}$ during $30 \mathrm{~min}$

2. Add $50 \mathrm{nM}$ Nocodazole or DMSO for control

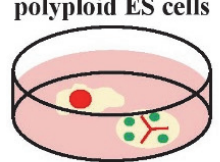

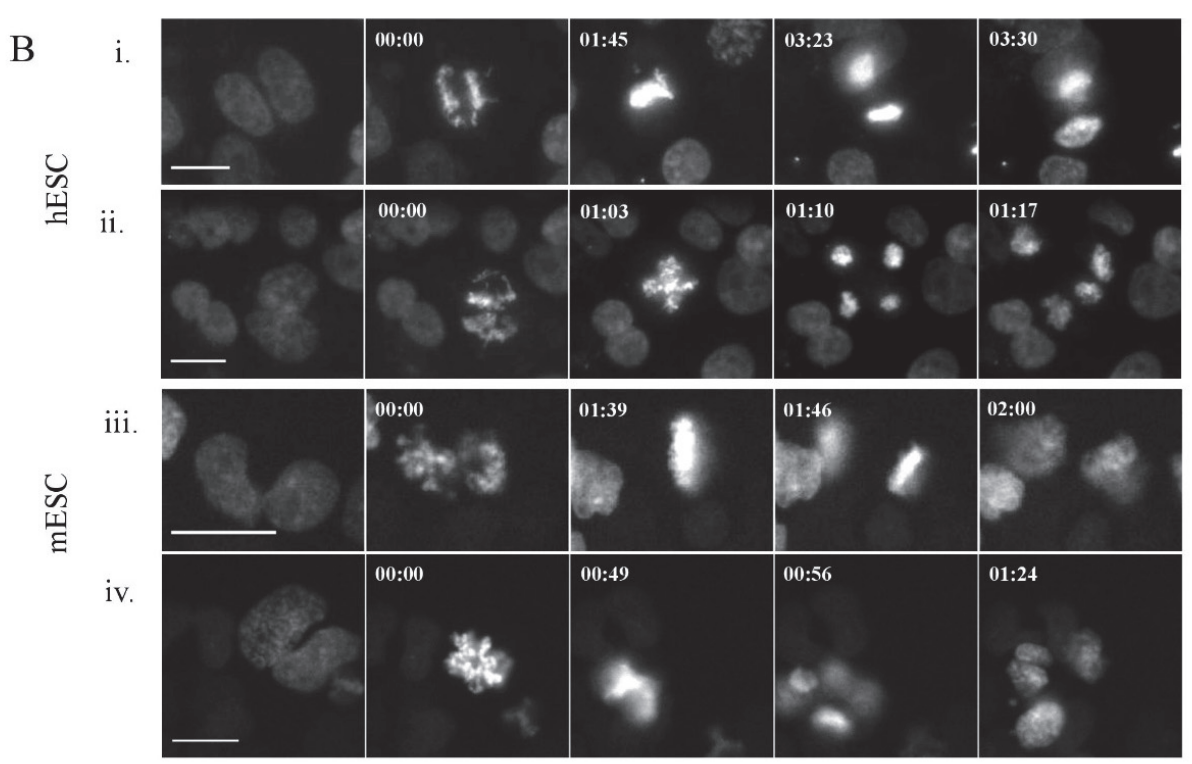

C

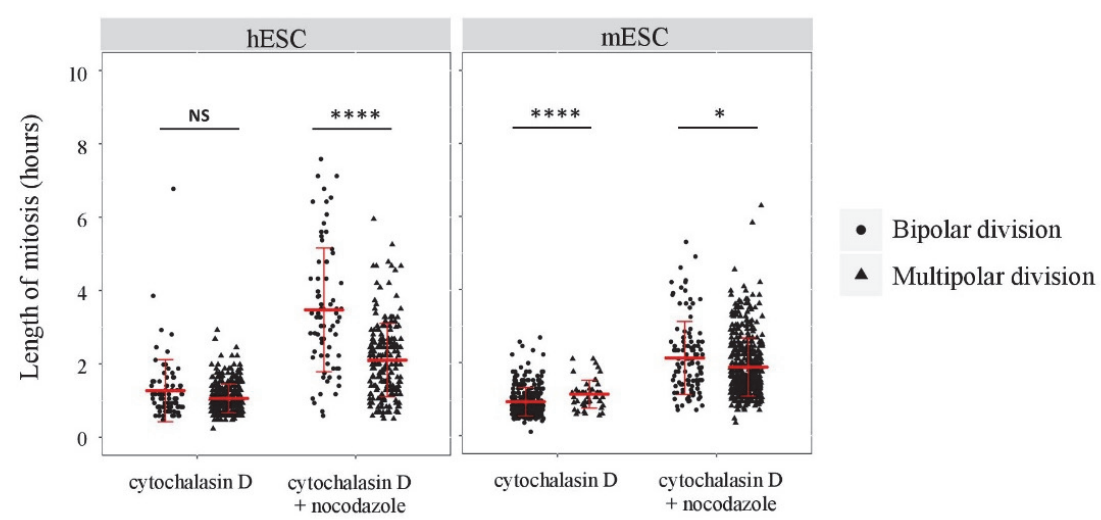

Fig. 5. Multipolar dividing human and mouse ES cells escape spindle assembly checkpoint (SAC) activity faster than bipolar dividing cells. (A) Experimental overview: To increase the number of polyploid cells, human and mouse ES cells were exposed to cytochalasin D for $12 \mathrm{~h}$ and then transferred into media containing $50 \mathrm{nM}$ nocodazole throughout imaging. Mitosis length was scored by live-cell imaging. (B) Representative images from the time-lapse experiments showing chromosome segregation during bipolar (i, iii) and multipolar (ii, iv) divisions in human and mouse ES cells. DNA was visualized by expression of histone H2A fused to EGFP, the relative time in minutes is indicated, scale bar $=20 \mu \mathrm{m}$. (C) Dot plots illustrating the mitosis length in polyploid human (left) and mouse (right) ES cells upon exposure to $50 \mathrm{nM}$ nocodazole. Mitosis length was scored in bipolar (dots) and multipolar (triangles) dividing cells. Control cells for both cell lines were treated with cytochalasin D treatment but not exposed to nocodazole. Data were obtained from three independent experiments. The numbers of scored cells in each category were 83, 354, 74, and 209 for human ES cells, and 307, 49, 111, and 500 for mouse ES cells. Statistical values are summarized by plotting the mean with error bars representing the standard deviation (red). Differences between groups were evaluated by Kruskal-Wallis test: n.s. $p>0.05,{ }^{*} p \leq 0.05,{ }^{* *} p \leq 0.01,{ }^{* * *} p \leq 0.001,{ }^{* * * *} p \leq 0.0001$.

Here, we evaluated both human and mouse ES cells and focused specifically on those undergoing multipolar divisions. Our data provided evidence that such cells are present in populations of undifferentiated human and mouse ES cells, although their frequency is normally fairly low. We also indirectly confirmed that the incidence of multipolar divisions in human ES cells correlated with the occurrence of cells with supernumerary centrosomes. Importantly, this suggests that the majority of ES cells with supernumerary centrosomes do not cluster centrosomes to avoid multipolar division. Clustering centrosomes, which are observed in many cancer cell lines, are not optimal as the daughter cells are often aneuploid or have dysregulated anaphase (Ganem et al., 2009). However, the lack of centrosome clustering might be caused by distinct p53 regulation in ES cells. Indeed, tetraploid p53/- cells have a much higher frequency of multipolar mitosis compared to 
their p53-expressing counterparts (Yi et al., 2011). Furthermore, p53 expression is upregulated upon ES cell differentiation and corresponds with that of Oct- 4 and Nanog (Lin et al., 2005; Zhang et al., 2014). This might explain why multipolar divisions are more frequent in undifferentiated ES cells. We previously showed that cells with supernumerary centrosomes are often tetraploid (Holubcova et al., 2011). In this study, blocking cytokinesis significantly increased the frequency of multipolar mitoses, which also correlated with an increased number of centrosomes accumulating after cytokinesis failure. The fact that tetraploid ES cells are viable indicates that they might have eliminated a mechanism involving the hippo pathway, which normally prevents proliferation of tetraploid cells (Ganem et al., 2014).

Our experiments indicate that mouse ES cells have lower number of multipolar mitoses in normal culture condition, suggesting that this phenomenon is more frequent in human than in rodent cells. Consistently with this, similar differences in errors of the spindle structure were also detected between human and mouse oocytes (Holubcova et al., 2015). Further it was demonstrated earlier that the percentage of supernumerary centrosomes substantially varies even between various hES cell lines and also with respect to their passage number (Holubcova et al., 2011). Therefore, more comprehensive studies using several human and mouse cell lines with different passage number would be required to draw firm conclusions on inter-species differences.

The cells resulting from multipolar divisions were not only viable, as our cell tracking results revealed, but they underwent subsequent divisions. Similar results were obtained using a glioblastoma cell line in which multipolar mitosis yielded viable and further dividing daughter cells (Gisselsson et al., 2010). The proliferation potential of such cells must be limited, as they would otherwise accumulate during extended proliferation in vitro. However, for potential stem cell therapies, such cells might still present a problem because they are inevitably aneuploid. Our data also showed that a subpopulation of multipolar dividing cells spent a longer time in interphase preceding this division. This indicated that the events prior to mitotic cell division might lead to aberrant spindle assembly and multipolar division.

We also evaluated whether the presence of multipolar division was monitored by cell cycle control mechanisms such as SAC, which is the ultimate mechanism that prevents chromosome segregation errors (Foley and Kapoor, 2013). Previous studies involving sea urchin eggs and somatic cells showed that SAC cannot postpone anaphase onset in cells with multipolar spindles (Sluder et al., 1997). However, more recent studies have shown that anaphase onset can be postponed in cancer cells with multipolar spindles, and their exit from mitosis is facilitated by mitotic slippage (Sluder et al., 1997). Our current results indicated that undifferentiated human and mouse ES cells possessed functional SAC that was capable of delaying anaphase upon exposure to a microtubule depolymerizing agent. However, the ability of SAC to permanently arrest cell cycling was limited, and cells eventually exited mitosis despite the presence of nocodazole. Importantly, our data suggested that ES cells of both species underwent multipolar divisions and were able to escape nocodazole arrest faster than cells with bipolar spindles. The ability of multipolar dividing ES cells to escape SAC control more efficiently resembles that seen in cancer cells and mammalian oocytes (Chen and Liu, 2015; Holubcova et al., 2015; Nakagawa and FitzHarris, 2017). In our study we employed fluorescence microscopy to track living cells. Using this technique, we were able to follow indi- vidual cells, which would be almost impossible using standard transmitted light/phase contrast illumination. However, fluorescence microscopy has also several drawbacks, such as the necessity to label intracellular structures by transgenes and possibility of a defects caused by the phototoxicity after prolonged experiments. Recently emerging technique of quantitative phase imaging (QPI), review in (Park et al., 2018) would allow to overpass the labelling and selection of fluorescently labelled cells and enabled to test more cell lines and make more general conclusions about multipolar divisions in ES cells.

\section{Conclusions}

Our data show for the first time that undifferentiated human and mouse ES cells can undergo multipolar division, and that their daughter cells are viable and can continue to divide. We also determined the frequency of multipolar divisions in undifferentiated mouse and human ES cells under standard culture conditions. Using cytochalasin D, a chemical inhibitor of cytokinesis, we demonstrated that the frequency of multipolar mitoses increased with polyploidization. We also showed for the first time that multipolar mitosis in ES cells changed the length of mitosis relative to that in cells with bipolar spindles. Cells resulting from multipolar divisions persisted in population for a relatively long time and engaged in subsequent divisions. Importantly, cells with multipolar spindles more quickly escaped from SAC activation-induced metaphase arrest. These results are important for determining the therapeutic potential of ES cells in cell therapy and tissue engineering. Our future studies will focus on cells with multipolar spindles during ES cell differentiation.

\section{Conflict of interests}

The authors declare no conflict of interests.

\section{Acknowledgements}

This work was supported by Czech Science Foundation projects 15-11707S and 15-04844S and the European Regional Development Fund-Project "National infrastructure for biological and medical imaging" (No. CZ.02.1.01/0.0/0.0/16_013/000 1775). We thank Drs. Anthony Hyman and Ina Poser (MPICBG, Dresden, Germany) for providing the H2A-GFP BAC construct, Dr. Ronald Naumann (MPI-CBG, Dresden, Germany) for providing the JM8.A mouse ES cells, and Dr. Klara Koudelkova for her excellent technical assistance.

\section{References}

Ballabeni A, Park IH, Zhao R, Wang W, Lerou PH, Daley GQ, Kirschner MW (2011). Cell cycle adaptations of embryonic stem cells. Proc Natl Acad Sci U S A 108(48): 19252-19257. DOI: 10.1073/pnas.1116794108.

Brinkley BR (2001). Managing the centrosome numbers game: from chaos to stability in cancer cell division. Trends Cell Biol 11(1): 18-21. DOI: 10.1016/s0962-8924(00)01872-9.

Castedo M, Perfettini JL, Roumier T, Valent A, Raslova H, Yakushijin K, et al. (2004). Mitotic catastrophe constitutes a special case of apoptosis whose suppression entails aneuploidy. Oncogene 23(25): 4362-4370. DOI: 10.1038/sj.onc.1207572.

Celton-Morizur S, Merlen G, Couton D, Margall-Ducos G, Desdouets $C$ (2009). The insulin/Akt pathway controls a specific cell division program that leads to generation of binucleated tetraploid liver cells in rodents. J Clin Invest 119(7): 1880-1887. DOI: $10.1172 /$ jci38677. 
Chen J, Liu J (2015). Erroneous silencing of the mitotic checkpoint by aberrant spindle pole-kinetochore coordination. Biophys J 109(11): 2418-2435. DOI: 10.1016/j.bpj.2015.10.024.

Draper JS, Smith K, Gokhale P, Moore HD, Maltby E, Johnson J, et al. (2004). Recurrent gain of chromosomes $17 \mathrm{q}$ and 12 in cultured human embryonic stem cells. Nat Biotechnol 22(1): 53-54. DOI: $10.1038 / \mathrm{nbt} 922$.

Duncan AW, Taylor MH, Hickey RD, Hanlon Newell AE, Lenzi ML, Olson SB, et al. (2010). The ploidy conveyor of mature hepatocytes as a source of genetic variation. Nature 467(7316): 707-710. DOI: 10.1038/nature09414.

Durrbaum M, Storchova Z (2016). Effects of aneuploidy on gene expression: implications for cancer. FEBS J 283(5): 791-802. DOI: 10.1111/febs.13591.

Foley EA, Kapoor TM (2013). Microtubule attachment and spindle assembly checkpoint signalling at the kinetochore. Nat Rev Mol Cell Biol 14(1): 25-37. DOI: 10.1038/nrm3494.

Galimberti F, Thompson SL, Ravi S, Compton DA, Dmitrovsky E (2011). Anaphase catastrophe is a target for cancer therapy. Clin Cancer Res 17(6): 1218-1222. DOI: 10.1158/1078-0432.CCR-101178.

Ganem NJ, Cornils H, Chiu SY, O'Rourke KP, Arnaud J, Yimlamai D, et al. (2014). Cytokinesis failure triggers hippo tumor suppressor pathway activation. Cell 158(4): 833-848. DOI: 10.1016/j. cell.2014.06.029.

Ganem NJ, Godinho SA, Pellman D (2009). A mechanism linking extra centrosomes to chromosomal instability. Nature 460(7252): 278-282. DOI: 10.1038/nature08136.

Gentric G, Desdouets C (2014). Polyploidization in liver tissue. Am J Pathol 184(2): 322-331. DOI: 10.1016/j.ajpath.2013.06.035.

Gisselsson D, Jin Y, Lindgren D, Persson J, Gisselsson L, Hanks S, et al. (2010). Generation of trisomies in cancer cells by multipolar mitosis and incomplete cytokinesis. Proc Natl Acad Sci U S A 107(47): 20489-20493. DOI: 10.1073/pnas.1006829107.

Holubcova Z, Blayney M, Elder K, Schuh M (2015). Human oocytes. Error-prone chromosome-mediated spindle assembly favors chromosome segregation defects in human oocytes. Science 348(6239): 1143-1147. DOI: 10.1126/science.aaa9529.

Holubcova Z, Matula P, Sedlackova M, Vinarsky V, Dolezalova D, Barta T, et al. (2011). Human embryonic stem cells suffer from centrosomal amplification. Stem Cells 29(1): 46-56. DOI: $10.1002 /$ stem.549.

Imreh MP, Gertow K, Cedervall J, Unger C, Holmberg K, Szoke K, et al. (2006). In vitro culture conditions favoring selection of chromosomal abnormalities in human ES cells. J Cell Biochem 99(2): 508-516. DOI: 10.1002/jcb.20897.

International Stem Cell Initiative, Amps K, Andrews PW, Anyfantis G, Armstrong L, Avery S, et al. (2011). Screening ethnically diverse human embryonic stem cells identifies a chromosome 20 minimal amplicon conferring growth advantage. Nat Biotechnol 29(12): 1132-1144. DOI: 10.1038/nbt.2051.

Kallas A, Pook M, Maimets M, Zimmermann K, Maimets T (2011). Nocodazole treatment decreases expression of pluripotency markers Nanog and Oct4 in human embryonic stem cells. PloS One 6(4): e19114. DOI: 10.1371/journal.pone.0019114.

Kwon M, Godinho SA, Chandhok NS, Ganem NJ, Azioune A, Thery M, Pellman D (2008). Mechanisms to suppress multipolar divisions in cancer cells with extra centrosomes. Genes Dev 22(16): 2189-2203. DOI: 10.1101/gad.1700908.

Lin TX, Chao C, Saito S, Mazur SJ, Murphy ME, Appella E, Xu Y (2005). P53 induces differentiation of mouse embryonic stem cells by suppressing Nanog expression. Nature Cell Biol 7(2): 165-171. DOI: $10.1038 / \mathrm{ncb} 1211$.

Martello G, Smith A (2014). The nature of embryonic stem cells. Annu Rev Cell Dev Biol 30: 647-675. DOI: 10.1146/annurevcellbio-100913-013116.
Nakagawa S, FitzHarris G (2017). Intrinsically defective microtubule dynamics contribute to age-related chromosome segregation errors in mouse oocyte meiosis-I. Curr Biol 27(7): 1040-1047. DOI: 10.1016/j.cub.2017.02.025.

Park Y, Depeursinge C, Popescu G (2018). Quantitative phase imaging in biomedicine. Nature Photonics 12(10): 578-589. DOI: 10.1038/s41566-018-0253-x.

Poser I, Sarov M, Hutchins JR, Heriche JK, Toyoda Y, Pozniakovsky A, et al. (2008). BAC TransgeneOmics: a highthroughput method for exploration of protein function in mammals. Nat Methods 5(5): 409-415. DOI: 10.1038/ nmeth.1199.

Quintyne NJ, Reing JE, Hoffelder DR, Gollin SM, Saunders WS (2005). Spindle multipolarity is prevented by centrosomal clustering. Science 307(5706): 127-129. DOI: 10.1126/ science.1104905.

Ring D, Hubble R, Kirschner M (1982). Mitosis in a cell with multiple centrioles. J Cell Biol 94(3): 549-556. DOI: 10.1083/jcb.94.3.549.

Sanchez-Aguilera A, Montalban C, de la Cueva P, SanchezVerde L, Morente MM, Garcia-Cosio M, et al. (2006). Tumor microenvironment and mitotic checkpoint are key factors in the outcome of classic Hodgkin lymphoma. Blood 108(2): 662-668. DOI: 10.1182/blood-2005-12-5125.

Santaguida S, Richardson A, Iyer DR, M'Saad O, Zasadil L, Knouse KA, et al. (2017). Chromosome mis-segregation generates cell-cycle-arrested cells with complex karyotypes that are eliminated by the immune system. Dev Cell 41(6): 638-651.e5. DOI: 10.1016/j.devcel.2017.05.022.

Silkworth WT, Cimini D (2012). Transient defects of mitotic spindle geometry and chromosome segregation errors. Cell Div 7(1): 19. DOI: 10.1186/1747-1028-7-19.

Silkworth WT, Nardi IK, Scholl LM, Cimini D (2009). Multipolar spindle pole coalescence is a major source of kinetochore misattachment and chromosome mis-segregation in cancer cells. Plos One 4(8): e6564. DOI: 10.1371/journal.pone.0006564.

Sluder G, Thompson EA, Miller FJ, Hayes J, Rieder CL (1997). The checkpoint control for anaphase onset does not monitor excess numbers of spindle poles or bipolar spindle symmetry. J Cell Sci 110(Pt 4): 421-429.

Spits C, Mateizel I, Geens M, Mertzanidou A, Staessen C, Vandeskelde Y, et al. (2008). Recurrent chromosomal abnormalities in human embryonic stem cells. Nat Biotechnol 26(12): 1361-1363. DOI: 10.1038/nbt.1510.

Storchova Z, Kuffer C (2008). The consequences of tetraploidy and aneuploidy. J Cell Sci 121(Pt 23): 3859-3866. DOI: 10.1242/ jcs.039537.

Vitale I, Galluzzi L, Castedo M, Kroemer G (2011). Mitotic catastrophe: a mechanism for avoiding genomic instability. Nat Rev Mol Cell Biol 12(6): 385-392. DOI: 10.1038/nrm3115.

Werbowetski-Ogilvie TE, Bosse M, Stewart M, Schnerch A, RamosMejia V, Rouleau A, et al. (2009). Characterization of human embryonic stem cells with features of neoplastic progression. Nat Biotechnol 27(1): 91-97. DOI: 10.1038/nbt.1516.

Yi Q, Zhao X, Huang Y, Ma T, Zhang Y, Hou H, et al. (2011). P53 dependent centrosome clustering prevents multipolar mitosis in tetraploid cells. PLoS One 6(11): e27304. DOI: 10.1371/journal. pone.0027304.

Zhang M, Cheng L, Jia Y, Liu G, Li C, Song S, et al. (2016). Aneuploid embryonic stem cells exhibit impaired differentiation and increased neoplastic potential. EMBO J 35(21): 2285-2300. DOI: $10.15252 / \mathrm{embj} .201593103$.

Zhang ZN, Chung SK, Xu Z, Xu Y (2014). Oct4 maintains the pluripotency of human embryonic stem cells by inactivating $\mathrm{p} 53$ through Sirt1-mediated deacetylation. Stem Cells 32(1): 157-165. DOI: $10.1002 /$ stem.1532. 